\title{
Generalized-Scattering-Matrix Extraction using the Finite-Volume Time-Domain (FVTD) Method
}

\author{
Dirk Baumann, Christophe Fumeaux, Pascal Leuchtmann, Rüdiger Vahldieck \\ Swiss Federal Institute of Technology (ETH) Zürich, IFH, 8092 Zürich, Switzerland
}

\begin{abstract}
In this paper the extraction of the generalized scattering matrix using the Finite-Volume Time-Domain (FVTD) method is presented. The extraction scheme takes advantage of the flux-splitting formulation that is inherent to the FVTD algorithm. Thus the incident and reflected waves in a transmission line are immediately known at every time step. A new way to determine the power waves is shown as well as the implementation of the ports and the mode separation in a multimode environment. The ability of the proposed scheme to extract the scattering parameters correctly is illustrated in an example that compares the simulated return loss of a balun to measurements.
\end{abstract}

Index Terms - FVTD, scattering parameters.

\section{INTRODUCTION}

The Finite-Volume Time-Domain (FVTD) method is applied to electromagnetic simulations since the end of the 1980s. Its ability to be utilized in unstructured meshes makes FVTD highly attractive to analyze complex problems with curved and oblique surfaces, high dielectric contrasts or small details embedded in a large overall structure [1].

Microwave components and devices are typically characterized by scattering matrices, making their extraction a recurrent and important problem in EM simulations. The computation of scattering matrices in guided wave structures with the FVTD method is not straightforward and encounters several difficulties. One of these difficulties has to do with the port definitions for which a solution has been proposed and successfully tested in [2].

This paper reports significant progress in the computation of the generalized scattering matrix by introducing a new method of calculating the power waves. Contrary to usual approaches the powers of the incident and reflected waves are determined directly by calculating the Poynting vectors of these waves in the port planes. The direct computation of the incident and the reflected power waves is possible because of the inherent separation of incoming and outgoing fluxes that the FVTD algorithm is based on. Template mode vectors are used to perform a modal projection to obtain the amplitude of the incident and reflected waves of the corresponding mode. The transversal field distributions stored in the mode vectors have to be determined in the preprocessing stage of the simulation.

To demonstrate the ability of the proposed scheme for accurate extraction of the scattering matrix, the simulation of a broadband balun that transforms the waves of an unbalanced coaxial cable to the waves of a balanced twowire line is shown and compared successfully to measurements.

\section{FVTD METHOD}

Maxwell's equations in conservative form are the foundation of the FVTD method. They are integrated over a finite volume using the divergence theorem. In our approach we use tetrahedrons as elementary cells in a cellcentered formulation. The tetrahedron $i$ with the volume $V_{i}$ consists of four faces $k_{i}$ having the area $F_{i k}$ with outward-pointing normal unit vectors $\boldsymbol{n}_{i k}$. For simplification the index $i$ will be left out in the following when denoting faces, face areas and normal vectors. The FVTD discretization of the Maxwell's equations in space and time is given by

$$
\begin{aligned}
\langle\boldsymbol{E}\rangle_{V_{i}}^{n+1} & =\langle\boldsymbol{E}\rangle_{V_{i}}^{n}+\frac{\Delta t}{\varepsilon V_{i}} \sum_{k=1}^{4}\left(n_{k} \times \overline{\langle\boldsymbol{H}\rangle_{F_{k}}}\right) F_{k} \\
\langle\boldsymbol{H}\rangle_{V_{i}}^{n+1} & =\langle\boldsymbol{H}\rangle_{V_{i}}^{n}-\frac{\Delta t}{\mu V_{i}} \sum_{k=1}^{4}\left(n_{k} \times \overline{\langle\boldsymbol{E}\rangle_{F_{k}}}\right) F_{k}
\end{aligned}
$$

where $\langle\cdot\rangle$ denotes spatially averaged values and the overbar averaged values over one time step $\Delta t$ [3]. On the left-hand side (LHS) in (1) volume-averaged fields at time-step index $n+1$ are obtained as a function of volume-averaged values at time step $n$ as well as surfaceand time-averaged fields. Our algorithm is second order in space using the MUSCL scheme. The Lax-Wendroff Predictor-Corrector scheme results in second-order accuracy in time [4].

The vector products on the right-hand side (RHS) of (1) are interpreted as mathematical fluxes through the tetrahedron's triangular faces. Only the tangential field 
components of the fields on the faces are relevant for the flux computation. These tangential fields on the triangles are used to determine the scattering parameters. A plane wave assumption in the finite volumes is made to separate the fluxes into an incoming and an outgoing part [4].

At each boundary between two cells the fluxes are written as

$$
\begin{aligned}
& \boldsymbol{n}_{k} \times \overline{\langle\boldsymbol{E}\rangle_{F_{k}}}=\boldsymbol{n}_{k} \times{\overline{\langle\boldsymbol{E}\rangle_{F_{k}}^{+}}}^{+}-\boldsymbol{n}_{k} \times \overline{\langle\boldsymbol{E}\rangle_{F_{k}}^{-}} \\
& \boldsymbol{n}_{k} \times \overline{\langle\boldsymbol{H}\rangle_{F_{k}}}=\boldsymbol{n}_{k} \times \overline{\langle\boldsymbol{H}\rangle_{F_{k}}^{+}}-\boldsymbol{n}_{k} \times \overline{\langle\boldsymbol{H}\rangle_{F_{k}}^{-}}
\end{aligned}
$$

The outgoing flux (superscript + ) through a cell face is estimated on the basis of barycenter field values in the considered cell.

The incoming flux (superscript - ) through a cell face is estimated on the basis of barycenter field values in the neighbor cell sharing the face.

In the following the denotation $\langle\cdot\rangle$ for spatially averaged fields and the overbar for temporally averaged fields will be left out for the sake of simplicity.

\section{GEOMETRICAL PORTS}

The ports in the FVTD algorithm are introduced as triangulated planes in the three dimensional (3D) tetrahedral mesh. The mesh generation is performed with the commercial software package Altair ${ }^{\circledR}$ HyperMesh $\circledast$. A port plane embedded in the mesh forces the adjacent tetrahedrons to have one face aligned, thus forming a phase reference plane.

For a port plane located on a boundary of the computational domain (plane 1 in Fig. 1), the incoming fluxes are used for excitation (source) or set to zero as a Silver-Müller absorbing boundary condition (ABC). A non-active port can alternatively be located in the computational bulk (plane 2 in Fig. 1), where it does not affect the FVTD algorithm.

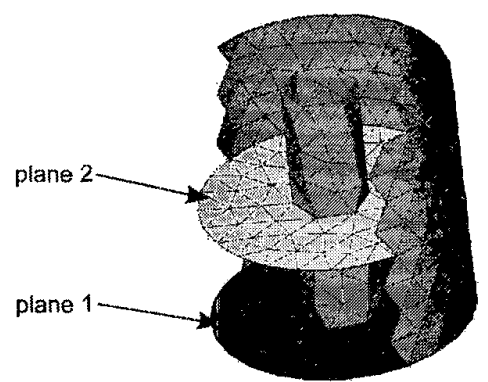

Fig. 1. The port planes are embedded in the inhomogeneous mesh of a coaxial cable. They can be either located on a boundary of the computational domain (plane 1) or in the computational bulk (plane 2). Volume elements are not shown.

\section{POWER WAVES}

To compute the scattering matrix, the incident and the reflected fields have to be determined on each port. In [5] Kurokawa introduced the concept of power waves. A new definition was given by Gwarek and Celuch-Marcysiak in [6] which guarantees that power waves also satisfy Maxwell's equations

$$
\begin{aligned}
& \breve{a}=\frac{\breve{V}+\breve{Z} \check{I}}{2 \sqrt{\check{Z}}}=\frac{\breve{V}^{+}}{\sqrt{\check{Z}}} \\
& \breve{b}=\frac{\breve{V}-\check{Z} \check{I}}{2 \sqrt{\check{Z}}}=\frac{\breve{V}^{-}}{\sqrt{\breve{Z}}}
\end{aligned}
$$

where the arc ${ }^{u}$ denotes frequency-domain quantities obtained by a Fourier transform and the superscripts + and - denote the incident and reflected waves respectively. Normalization with the characteristic impedance $\bar{Z}$ of the transmission line results in a generalization of the scattering parameters [7]. Unfortunately, the application of this definition requires to compute the voltage $\breve{V}$, the current $\breve{I}$, and the characteristic impedance of the transmission line $\breve{Z}$.

A new approach to determine the power waves satisfying Maxwell's equations is proposed here for the FVTD method. The powers of the incident and reflected waves are obtained by integration of the Poynting vectors over the port cross section at time step $n$. None of the magnitudes $\breve{V}, \breve{I}$ and $\breve{Z}$ need to be computed. On the spatially discretized port plane consisting of $N$ triangles, where the triangle $k$ has the area $F_{k}$, these integrals are approximated as sums

$$
\begin{gathered}
a(n)^{2}=\sum_{k=1}^{N}\left(\boldsymbol{E}_{t}^{+}(k, n) \times \boldsymbol{H}_{t}^{+}(k, n)\right) \boldsymbol{F}_{k} \\
b(n)^{2}=\sum_{k=1}^{N}\left(\boldsymbol{E}_{t}^{-}(k, n) \times \boldsymbol{H}_{t}^{-}(k, n)\right) \boldsymbol{F}_{k}
\end{gathered}
$$

Because of the local plane wave assumption the magnetic field of the separated waves can be expressed as

$$
\boldsymbol{H}_{t}^{+1-}=\frac{\boldsymbol{n} \times \boldsymbol{E}_{t}^{+/-}}{\eta}
$$

with $n$ being the outward pointing unit normal vector of the considered face and $\eta$ the wave impedance in the material surrounding the port plane. The tangential components $\boldsymbol{E}_{t}^{+/-}$and $\boldsymbol{H}_{t}^{+/-}$of the incident and reflected waves are known from the flux-splitting scheme in FVTD. Frequency domain values of the incident and 
reflected waves are obtained by carrying out a discrete Fourier transformation on the fly during the march-in-time iteration.

\section{MODE PROJECTION}

In a transmission line multiple modes may exist in one geometrical port. Each of these modes is assigned to one electromagnetic port $m$, i.e. a geometrical port can hold several electromagnetic ports. To determine multimode scattering parameters, a modal extraction must be performed in each geometrical port plane.

The simulated total electric and magnetic field on the geometrical port triangle $k$ at time step $n$ can be expressed as a sum over all possible modes on that port

$$
\begin{aligned}
& \boldsymbol{E}_{t}^{+/-}(k, n)=\sum_{m} A_{m}^{+/-}(n) \boldsymbol{e}_{m}(k) \\
& \boldsymbol{H}_{t}^{+/-}(k, n)=\sum_{m} A_{m}^{+/-}(n) \boldsymbol{h}_{m}(k)
\end{aligned}
$$

where $\boldsymbol{e}_{m}(k)$ and $\boldsymbol{h}_{m}(k)$ are normalized template mode vectors containing the analytically obtained transversal field distribution of the mode $m \cdot A_{m}^{+1-}(n)$ is the corresponding mode amplitude.

In order to separate the components of the field of a certain mode $m$ from the total field in the port, the tangential components of the simulated field are projected onto the orthogonal template mode vector [8]

$$
A_{m}^{+/-}(n)=\sum_{k=1}^{N} \boldsymbol{E}_{t}^{+/-}(k, n) \cdot \boldsymbol{e}_{m}(k) F_{k}
$$

where $\boldsymbol{E}_{t}^{+/-}$is the numerical result of the incident/ reflected tangential electric field in the port plane obtained in the FVTD simulation.

The power waves $a_{m}(n)$ and $b_{m}(n)$-corresponding to (5) and (6) - associated with mode $m$ are determined using the calculated mode amplitude $A_{m}^{+/-}(n)$ and the mode template vectors

$$
\begin{aligned}
& \boldsymbol{E}_{m, t}^{+/-}(k, n)=A_{m}^{+/-}(n) \cdot \boldsymbol{e}_{m}(k) \\
& \boldsymbol{H}_{m, t}^{+/-}(k, n)=A_{m}^{+/-}(n) \cdot \boldsymbol{h}_{m}(k)
\end{aligned}
$$

In a mono-mode environment the mode projection helps to reduce the number of cells in a port plane that are needed for convergence of the scattering parameters. Hence the mode projection saves computational cost. Nevertheless the cell sizes in the port region remain typically small in comparison to the element size of the rest of the computational domain. Consequently the port cells are crucial for determining $\Delta t$. The restraint to use $\Delta t$ in the whole computational domain is abolished by the geometry- matched local time step scheme that was introduced in [3]. The local time steps allow a significant speed-up of the simulation by using a short time step in the port region and longer time steps where larger cells exist.

\section{RESULTS}

To demonstrate the ability of the proposed scheme to accurately compute the scattering parameters, the return loss of a broadband balun is simulated using FVTD. The simulation results are compared to measurements. The balun transforms waves from an unbalanced coaxial cable to a balanced two-wire line. For example this balun can be applied in the feeding structure of an antenna, where it additionally transforms the $50 \Omega$ impedance of the coaxial cable to the antenna impedance. Figure 3 shows a 3D view of the balun model. Basically the balun consists of a coaxial cable that feeds a balanced paired-strip line ending in a two-wire line. The center conductor of the coaxial cable is lead through a hole in the substrate of the pairedstrip line and is terminated with a stub as displayed in the inset of Fig. 3.

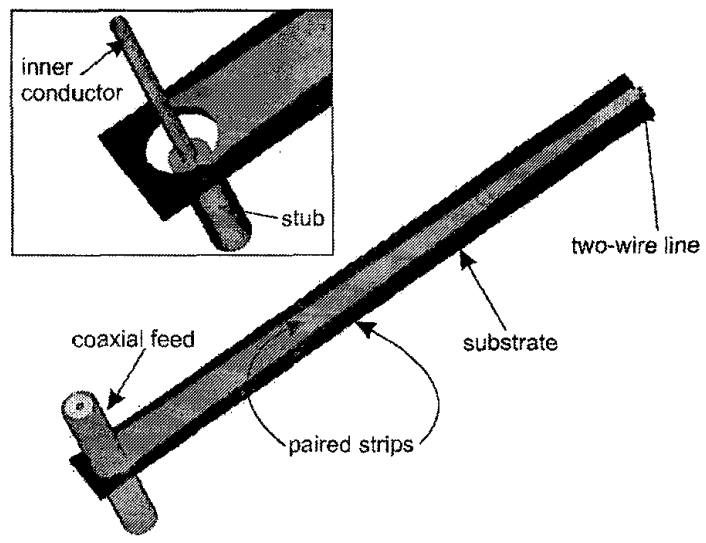

Fig. 3. Detailed model of the balun. The inset shows the feed through the substrate of the inner conductor that is terminated in a stub.

The stub provides a symmetrical transition from the coaxial cable to the paired-strip line. The length of the stub is approximately a quarter wavelength at the center frequency. The outer conductor of the coaxial cable is connected on both sides to the paired-strip line and to a cavity that is surrounding the feed section (not shown in Fig. 3). The magnified view of the coaxial feed on the LHS of Fig. 4 shows that the thickness of the outer conductor is taken into account in the model. The port plane is located at the end of the cable. 

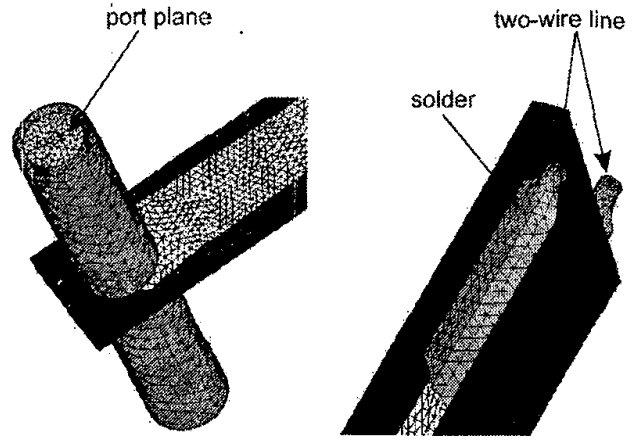

Fig. 4. Balun details with surface discretization. LHS: Coaxial feeding section containing the port plane. RHS: The top part of the balun forms the transition between paired strips and a twowire line that is soldered to the strips.

The paired-strip line is tapered along the balun length in order to provide the impedance transformation. The transition of the paired strips to the two-wire line is shown in detail on the RHS of Fig. 4 that displays the soldering of the two-wire line to the paired strips. In the FVTD simulation the two-wire line is terminated with an $A B C$.

The balun is investigated in the broadband frequency range of 1 to $20 \mathrm{GHz}$. In Fig. 5 the simulated result of $S_{11}$ is compared to measurement results.

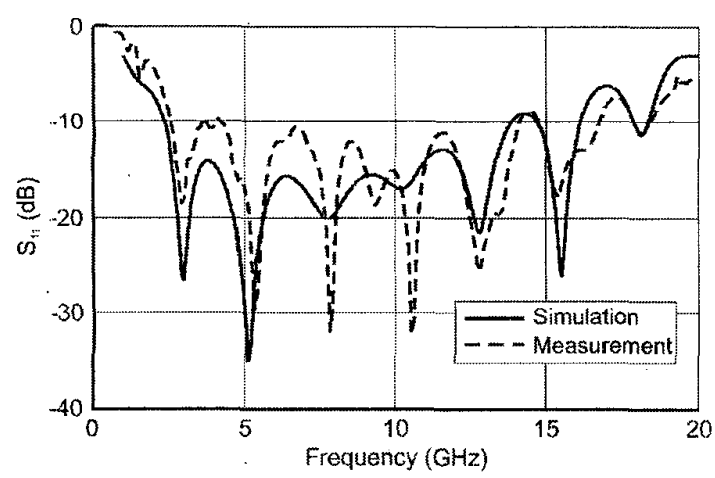

Fig. 5. Comparison between the simulated and measured $S_{11}$ of the balun.

The overall agreement is good. The rippled structure of the measurement is resolved in the simulation results. The origin of discrepancies around $8 \mathrm{GHz}$ is believed to be caused by the different (imperfect) matching conditions at both ends of the balun in the simulation and the measurement. The bandwidth for practical operation of the balun obtained in the simulation is matching the measured per- formance, e.g. a $-10 \mathrm{~dB}$ bandwidth is achieved between 4 and $12 \mathrm{GHz}$.

\section{CONCLUSION}

In this paper a novel scheme to extract the generalized scattering matrix for the FVTD method is proposed. The flux-splitting formulation, inherent to the FVTD algorithm, is exploited to obtain the incident and the reflected fields on a port plane at each time step. These fields are projected on the modes $m$ associated with this port to obtain multimode scattering parameters. To demonstrate the capability of the proposed scheme, the reflection coefficient of a balun is simulated and compared with good agreement to measured results.

\section{ACKNOWLEDGEMENT}

The authors wish to acknowledge the support of the Swiss Defense Procurement Agency (Armasuisse), Bern.

\section{REFERENCES}

11 D. Baumann, C. Fumeaux, P. Leuchtmann, R. Vahldieck, "Finite-Volume Time-Domain (FVTD) modeling of : broadband double-ridged horn antenna", to be published in Int. J. Numer. Model., March/April 2004

12| D. Baumann, C. Fumeaux, P. Leuchtmann, R. Vahldieck "Finite-Volume Time-Domain (FVTD) method and its application to the analysis of hemispherical dielectricresonator antennas", IEEE MTT-S Int. Microwave Symp. Dig., Philadelphia, PS, pp. 985-988, June 2003.

13| C. Fumeaux, D. Baumann, P. Leuchtmann, R. Vahldieck, "A generalized local time-step scheme for efficient FVTD simulations in strongly inhomogeneous meshes", to be published in IEEE Trans. Microwave Theory \& Tech., vol. 52, no. 3, March 2004

141 S. M. Rao, Time domain electromagnetics, Chapter 9: Finite-Volume time domain method, Academic Press, 1999

$|5|$ K. Kurokawa, "Power waves and the scattering matrix", IEEE Trans. Microwave Theory \& Tech., vol. 13, no. 3, pp. 194-202, March 1965.

|6| W. K. Gwarek, M. Celuch-Marcysiak, "Wide-band Sparameter extraction from FD-TD simulations for propagating and evanescent modes in inhomogeneous guides, IEEE Trans. Microwave Theory \& Tech., vol, 51, no. 8, pp. 1920-1928, August 2003.

17| D. M. Pozar, Microwave engineering, Addison-Wesley, 1990.

[8] E. A. Navarro, T. M. Bardallo, J. Navasquillo-Miralles, "FDTD characterization of evanescent modes - multimode analysis of waveguide discontinuities", IEEE Trans. Microwave Theory \& Tech., vol. 48, no. 4, pp. 606-610, April 2000 